\title{
Influence of Many-Body Effects in Real Metals on Electron-Positron Momentum Distributions
}

\author{
E. BOROŃSKI*, \\ Institute of Physics, Opole University, Oleska 48, 45-052 Opole, Poland
}

\begin{abstract}
It is shown that if one takes into account the effective mass of the electron, which in real structures is actually different than the free electron mass, the electron and positron self-energy effects may result in flatter and smaller enhancement of the electron-positron momentum distribution. Thus, the many-body effects mentioned above, among other reasons like e.g. influence of lattice potential on electron and positron wave functions, can be responsible for decreasing of the discontinuity on the Fermi momentum and a greater smearing of the Fermi surface seen in several angular correlation of positron annihilation radiation experiments.
\end{abstract}

DOI: $10.12693 /$ APhysPolA.125.706

PACS: $78.70 . \mathrm{Bj}, 71.18 .+\mathrm{y}, 71.60 .+\mathrm{z}, 71.15 . \mathrm{Mb}, 71.10 . \mathrm{Ca}$

\section{Introduction}

Angular correlation of positron annihilation radiation (ACPAR) is one of important methods of investigating the electronic structure of solids $[1,2]$. These investigations are usually supported by theoretical calculations which, by applying advanced methods, help in the interpretation of the results $[1,3]$. These studies base, however, on the old but commonly accepted result of calculations of Carbotte and Kahana [4], that the momentum distributions which result from dynamical electron electron (e-e) and electron-positron (e-p) interactions, due to some cancellations, can be neglected above the Fermi surface.

The hitherto applied theoretical methods cannot, unfortunately, take at the same time into consideration all many-body effects and the crystal-lattice potential. Thus, these methods base on enhancement factors describing an increase of electron density due to the $\mathrm{e}-\mathrm{p}$ interaction, applied mostly in the local density approximation [5]. The recognized attempt to include lattice effects into the e-p interaction was the Bloch modified ladder approach [6]. This approach is, however, not self-consistent and neglects dynamic e-e and e-p interactions. Moreover, the calculations are very tedious and requiring a lot of computational time [6].

In last years some relatively complicated Fermi surfaces have been studied experimentally by angular correlation of annihilation radiation and interpreted with advanced formalisms $[3,7,8]$. Interestingly, some results indicated a similarity to data yielded from the Compton experiments, characterized usually by high smearing of the Fermi surface emerging due to strong electronelectron correlations and considerable tails. A possibility of occurring tails have been considered by Manuel et al. [9] when interpreting ACPAR curves. In turn, the ex-

\footnotetext{
*e-mail: e.boronski@uni.opole.pl
}

traordinary smearing of the Fermi surface was attributed $[3,10]$ to a considerable contribution of the e-e correlations in electron-positron momentum distributions.

The attempt to explain whether these many-body tails have to vanish in real structures as well as in the case of the positron in the electron gas has been undertaken in Ref. [11]. Following Carbotte and Kahana [4], within the Green function formalism to the first order, the authors showed that for effective masses of electrons greater than free electron mass the contributions from dynamic $\mathrm{e}-\mathrm{p}$ interaction and electron (e.s.e.) and positron self-energy (p.s.e.) effects do not enough effectively cancel each other. Therefore the e-p distributions change and the tails cannot be negligible.

Since the previous calculations have been made to the first order in perturbation series, in this paper one tries to correct the results by multiplying them by an appropriate enhancement factor which is found in the ladder approximation to the $\mathrm{e}-\mathrm{p}$ scattering amplitude. The calculations are performed on the base of the Bethe-Goldstone-like equation at the assumption that the effective mass of the electron can differ from the free electron mass and that electrons may scatter with some probability into the region of momenta $p<p_{\mathrm{F}}$ as well as to $p>p_{\mathrm{F}}[12,13]$. The final influence of the self-energy effects for different values of electron effective masses on the momentum distributions for $p<p_{\mathrm{F}}$ and $p>p_{\mathrm{F}}$ and the possible consequences on the smearing of the Fermi surfaces are shown in Sect. 3.

\section{Theory}

The momentum distribution of the annihilating $\mathrm{e}-\mathrm{p}$ pair can be presented in terms of the two-particle Green function [4]:

$$
\begin{aligned}
& \rho(\boldsymbol{p})=(\mathrm{i})^{2} \int \mathrm{d}^{3} \boldsymbol{x} \mathrm{d}^{3} \boldsymbol{y} \exp (-\mathrm{i} \boldsymbol{p} \cdot(\boldsymbol{x}-\boldsymbol{y})) \\
& \quad \times G_{\mathrm{ep}}\left(\boldsymbol{x} t, \boldsymbol{x} t ; \boldsymbol{y} t^{+}, \boldsymbol{y} t^{+}\right) .
\end{aligned}
$$

The partial annihilation rate $R(\boldsymbol{p})$ for the total momentum $\boldsymbol{p}$ of the e-p pair is related to $\rho(\boldsymbol{p})$ simply by $\lambda / \Omega \times \rho(\boldsymbol{p})$, where $\lambda$ is the relation of the annihilation rate 
for the positronium in its singlet state to the positronium density, and $\Omega$ is the volume of the crystal. Within the ladder approximation (Fig. 1) the $G_{\mathrm{ep}}\left(\boldsymbol{x} t, \boldsymbol{x} t ; \boldsymbol{y} t^{+}, \boldsymbol{y} t^{+}\right)$ can be determined by the Bethe-Goldstone amplitude which corresponds to electron-positron wave function that has been found by many authors for the electron gas [14-16]. For the positron in the electron gas it yields the enhancement factor $\varepsilon(p)$ which describes how the probability density of unperturbed electronic states changes due to $\mathrm{e}-\mathrm{p}$ interaction. For real metals one often approximates the two-body wave function by a product of the Slater determinant of one-electron orbitals $\phi_{\boldsymbol{k}}^{i}(\boldsymbol{r})$, the positron wave function $\phi_{+}(\boldsymbol{r})$ and a factor which is, in general, a complicated functional of electron and positron wave function but approximately can be linked to an enhancement factor $\varepsilon$, in e.g. the LDA approximation, by the relation

$$
\gamma^{2}=\varepsilon
$$

Then $R(\boldsymbol{p})$ reads

$$
\begin{aligned}
R(\boldsymbol{p}) & =\frac{\lambda}{\Omega} \sum_{\boldsymbol{k}, i} n_{\boldsymbol{k}, i} \\
\times & \left|\int \mathrm{e}^{-\mathrm{i} \boldsymbol{p} r} \gamma\left(\boldsymbol{k}, i, r_{\mathrm{s}}(\boldsymbol{r})\right) \phi_{\boldsymbol{k}}^{i}(\boldsymbol{r}) \phi_{\mathbf{0}}^{+}(\boldsymbol{r}) \mathrm{d} \boldsymbol{r}\right|^{2},
\end{aligned}
$$

where $\boldsymbol{p}$ is the wave vector corresponding to the total momentum of interacting e-p pair, $\boldsymbol{k}$ is the wave vector of the electron undisturbed by the positron and $i$ is the band index. The values of the occupation number $n_{\boldsymbol{k}, i}$ may differ from 1 only due to lattice or temperature effects.

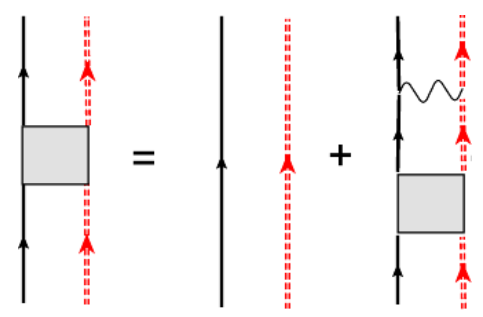

Fig. 1. The ladder approximation leading to the Bethe-Goldstone equation [13, 14]. Solid line - electron propagator, double dashed line - positron propagator. The horizontal dashed line represents the screened static RPA interaction.

In general, the two-particle propagator in Eq. (1) corresponds to an infinite series of the Feynman diagrams. This expansion, in particular, consists of terms with electron and positron self-energy and $\mathrm{e}-\mathrm{p}$ interaction contributions, and the $\mathrm{e}-\mathrm{e}$ and $\mathrm{e}-\mathrm{p}$ potentials are dynamic. Certainly, the approximation (3) does not take into account that each particle can interact with itself through the polarizable medium, moreover the potential necessary to find $\gamma\left(\boldsymbol{k}, i, r_{\mathrm{s}}(\boldsymbol{r})\right)$ in (3) is mostly static. This kind of approximation has been, however, commonly used for its simplicity. The next argument for using the above approach comes from Carbotte and Kahana [4] who have shown for the positron in the electron gas that replacing the dynamic potential by static one only weakly changes the $\mathrm{e}-\mathrm{p}$ momentum distribution for $p<p_{\mathrm{F}}$, and for $p>p_{\mathrm{F}}$ the values of this distribution are negligible owing to an effective cancellation of self-energy and the dynamic e-p interaction contributions. This cancellation applies also in higher orders of perturbation series.

Can the reduction of the mentioned effects be so effective for real metals? The approach proposed in Ref. [11] and the results contradict this statement. The authors propose a simple model in which an electron (eventually the positron) in a real metallic structure can be characterized by an appropriate effective mass, different from the free electron mass. Then, in a free electron and positron propagators

$$
\begin{gathered}
G_{\mathrm{e}}^{0}(\boldsymbol{q} ; \omega)=\frac{\theta\left(k_{\mathrm{F}}-|\boldsymbol{q}|\right)}{\hbar^{2} q^{2} / 2 m_{\mathrm{e}}-\omega+\mathrm{i} 0^{+}} \\
+\frac{\theta\left(|\boldsymbol{q}|-k_{\mathrm{F}}\right)}{\hbar^{2} q^{2} / 2 m_{\mathrm{e}}-\omega-\mathrm{i} 0^{+}}, \\
G_{\mathrm{p}}^{0}(\boldsymbol{q} ; \omega)=\frac{\theta(-q)}{\hbar^{2} q^{2} / 2 m_{\mathrm{e}}-\omega+\mathrm{i} 0^{+}} \\
+\frac{\theta(q)}{\hbar^{2} q^{2} / 2 m_{\mathrm{e}}-\omega-\mathrm{i} 0^{+}},
\end{gathered}
$$

the corresponding free electron (positron) mass is replaced by an effective one

$$
\begin{aligned}
& m_{\mathrm{e}} \longrightarrow m_{\mathrm{e}} m_{\mathrm{e}}^{*}, \\
& m_{\mathrm{p}} \longrightarrow m_{\mathrm{e}} m_{\mathrm{p}}^{*} .
\end{aligned}
$$

On this basis the dynamic e-e and e-p potentials are determined within the RPA approximation and then the e.s.e. and p.s.e. contributions (Fig. 2) are calculated. As shown [11], the mutual reduction of these contributions is weaker if the effective mass of the electron increases and the many-body tails in momentum distributions are no more negligible. This can confirm the hypothesis [10] on the meaning of dynamical correlations in $\mathrm{e}-\mathrm{p}$ momentum distributions.
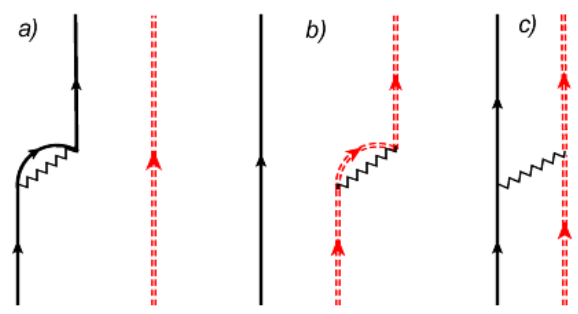

Fig. 2. The diagrams represent the first order electron (solid line) and positron (double dashed line) contributions to momentum dependent annihilation rate: (a) electron self-energy correction, (b) positron self-energy correction, (c) electron-positron ladder diagram. The frequency dependent RPA potential is used as the dynamic interaction (zigzag line). 
As mentioned, the calculations $[4,11]$ have been performed for the first order diagrams. The approach can be improved by multiplying [4] the first order terms in the expression for the e-p momentum distribution by an enhancement factor $\varepsilon(p)$. Thus, the $\mathrm{e}-\mathrm{p}$ momentum distribution $\rho(p)$ could be approximated as

$$
\rho(p)=\left[\rho^{\text {e.s.e. }}(p)+\rho^{\text {p.s.e. }}(p)+\rho^{(1)}(p)\right] \varepsilon(p),
$$

where $\varepsilon(p)$ is the enhancement factor $\left(\rho^{(1)}(p)\right.$ stands for the first order dynamic ladder correction to the $\rho(p))$. This means that instead of full perturbation expansion of $R(p)$ with dynamic potential and self-energy effects in all orders, the infinite series of ladders (with a static potential) is added to the first order diagrams in which the self-energy effects and e-p interaction are described by the dynamic RPA potential. The enhancement factors calculated according to such a scheme should not be limited to the range of $p<p_{\mathrm{F}}$ (in general this quantity can be defined for the whole regime of electron momenta, however, the approximation used by Kahana [14] limited it to the region $p<p_{\mathrm{F}}$ ). The approach presented in Ref. [12] fulfills this requirement. It uses the BetheGoldstone-like equation for the thermalized positron and the electron in the initial state $\boldsymbol{p}$ :

$$
\begin{aligned}
& \chi(\boldsymbol{k}, \boldsymbol{p})=\frac{1}{\Omega} \frac{1}{\frac{\hbar^{2}}{2 m_{\mathrm{e}}}\left[k^{2}+(\boldsymbol{k}-\boldsymbol{p})^{2}-p^{2}\right]} \\
& \times\left\{u(|\boldsymbol{k}-\boldsymbol{p}|, 0)+\sum_{\boldsymbol{q}}[1-n(\boldsymbol{q})] u(|\boldsymbol{q}-\boldsymbol{p}|, 0) \chi(\boldsymbol{q}, \boldsymbol{p})\right\} .
\end{aligned}
$$

In Eq. (8) some self-energy effects are [12, 13], though approximately, included in all orders, allowing electrons to be scattered with some probability to any states below and above the Fermi surface, and excluding the case when $\boldsymbol{k}=\boldsymbol{p} . \quad u(|\boldsymbol{k}|, 0)$ is the e-p interaction in static RPA approximation. $n_{k}$ are the occupation numbers (corresponding to the Daniel and Vosko distribution [17]) calculated within the RPA approximation.

Then the corresponding formula for $\gamma\left(p ; r_{\mathrm{s}}\right)$ reads

$$
\gamma\left(p ; r_{\mathrm{s}}\right)=1+\sum_{\boldsymbol{k}}\left(1-n_{\boldsymbol{k}, r_{\mathrm{s}}}\right) \chi\left(\boldsymbol{k}, \boldsymbol{p} ; r_{\mathrm{s}}\right)
$$

and, with the use of (2), the momentum dependent enhancement factor can be calculated from (9).

In our effective mass model one replaces the denominator in (8) by the following expression:

$$
\frac{\hbar^{2} k^{2}}{2 m_{\mathrm{e}}^{*}}+\frac{\hbar^{2}(\boldsymbol{k}-\boldsymbol{p})^{2}}{2 m_{\mathrm{p}}^{*}}-\frac{\hbar^{2} p^{2}}{2 m_{\mathrm{e}}^{*}}
$$

in which the free electron and positron mass has been replaced by the appropriate effective masses. Respectively, the occupation numbers $n(\boldsymbol{q})$ and the e- $\mathrm{p}$ interaction $u(|\boldsymbol{q}-\boldsymbol{p}|, 0)$ have been changed. The calculation procedure requires also that the singularities for $\boldsymbol{k}=\boldsymbol{p}$ have to be taken into account. This is solved in the analogous way as in Ref. [12], but the fraction $k / p$ in the Legendre functions in the formula (13) of Ref. [12] is replaced by $\left(k^{2}+p^{2}\right) / 2 k p$ and the integral in this formula is additionally multiplied by $m_{\mathrm{e}}^{*} / m_{\mathrm{p}}^{*}$. The procedure de- scribed above yields for different electron effective masses the enhancement factors as well as total $\mathrm{e}-\mathrm{p}$ momentum distributions to all orders in perturbation series.

\section{Results}

According to Eqs. (8), (9) and (2) we have found the enhancement factors corresponding to electron density parameter $r_{\mathrm{s}}=2$ and various effective masses of the electrons: $m_{\mathrm{e}}^{*}=1, m_{\mathrm{e}}^{*}=5, m_{\mathrm{e}}^{*}=10($ Fig. 3$)$. Of course, in principle this is not a trivial matter to accept any realistic values for the effective masses, as they are tensors and many effects contribute to, however, to estimate the effect [11] one could take some known values for $m_{\mathrm{e}}^{*}$ from e.g. Table (2.3) of Ashcroft and Mermin book [18]. The results for $m_{\mathrm{e}}^{*}=1$ presented [12] for $p<p_{\mathrm{F}}$ resemble the ones calculated by Kahana and later authors, and their dominant feature is a strong momentum dependence. For $p>p_{\mathrm{F}}$ the values of the enhancement dynamically go down as the cross-section for scattering of two particles decreases with their velocity. For greater masses, however, the curves become lower than for $m_{\mathrm{e}}^{*}=1$ and quite flat. This means that the $\mathrm{e}-\mathrm{p}$ interaction is not strong enough to change the density of heavier electrons in its neighbourhood.

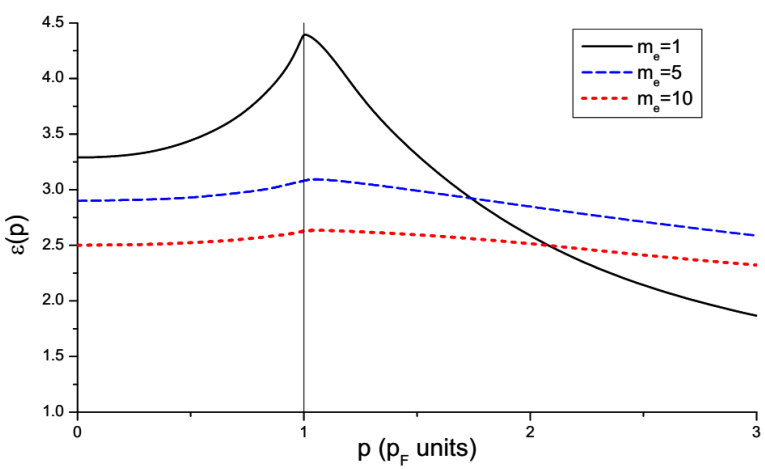

Fig. 3. The enhancement factor for $r_{\mathrm{s}}=2$ and different effective masses of electrons, calculated on the base of Bethe-Goldstone equation in which electron self-energy effects are approximately taken into account [13], solid line.

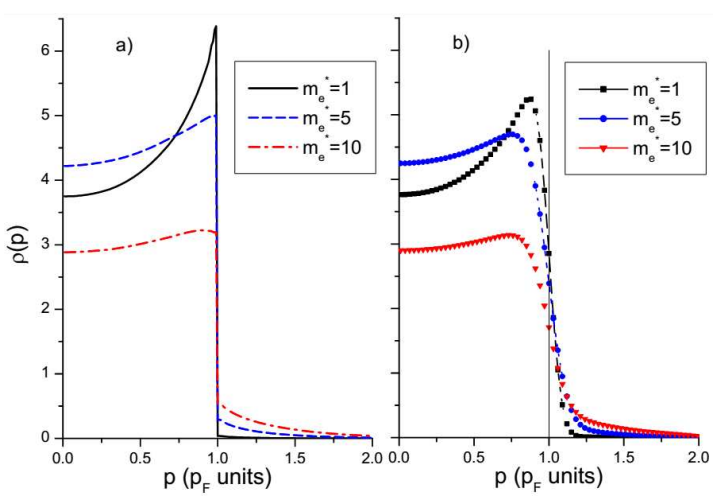

Fig. 4. The momentum distribution for $r_{\mathrm{s}}=2$ and different effective masses of electrons (a), and smeared with the resolution function of FWHM $=0.1$ (b). 
The momentum distributions, as results of product of the enhancement factors by the sum of first order terms, according to Eq. (7), are presented in Fig. 4a. For $m_{\mathrm{e}}^{*}=1$ there are only few electrons possessing enough high momenta to be above the Fermi surface, so the total curve corresponding to the momentum distribution still breaks rapidly at $p_{\mathrm{F}}$ and the high momentum tail is negligible (Fig. 4a), confirming the old result of Carbotte and Kahana [4] and the recent Monte Carlo result of Drummond et al. [20]. However, for greater effective masses the tails substantially grow up. At the same time the distributions below $p_{\mathrm{F}}$ become more flat and the difference is evidently seen near the Fermi momentum. What is more, for $m_{\mathrm{e}}^{*}=10$ (and possibly for bigger masses) one can observe that below $p_{\mathrm{F}}$ the values of the distribution function considerably decrease. Thus, in general, the discontinuity at the Fermi momentum decreases when increasing the values of the effective mass of the electrons. In order to see how the resolution function of the measuring devices could influence the presented results, we have additionally calculated the convolution of the $\mathrm{e}-\mathrm{p}$ momentum distribution functions with the Gaussian of $\mathrm{FWHM}=0.1$, so the corresponding curves have been smeared off. The results are presented in Fig. 4b. The visible tails corresponding to $m_{\mathrm{e}}^{*}>1$ are quite substantial and can be observed up to $2 p_{\mathrm{F}}$.

\section{Conclusions}

The improvements presented in the second part of Sect. 2 allow for more realistic estimation of the influence of the self-energy effects and different electron effective masses on the e-p momentum distributions. The probability that the electron of the momentum $p>p_{\mathrm{F}}$ can be scattered to lower or higher states is quite high. Certainly, the number of such electrons is very low for $r_{\mathrm{s}}=2$ and for the effective mass equal to the mass of a free electron, however if one assumes that this mass can be higher, then owing to the self-energy effects, the number of these electrons grows up and the effect becomes meaningful. It leads to arising of substantial many-body tail in momentum distributions for high momenta. The self-energy effects change also the distributions below the Fermi momentum. In particular, for higher effective masses of electrons these distributions become more flat and enhancements are, in general, lower than those calculated for $m_{\mathrm{e}}^{*}=1$. In general, when taking into account higher effective electron masses the effect of dynamic correlations on theoretical e-p momentum distributions become similar to those observed in the Compton experiments. Thus, as was shown in the previous section, the self-energy effects in real metallic structures may lead to some extra smearing of the experimentally seen Fermi surface. This fact can be quite important for the interpretation of the experimental data.

\section{Acknowledgments}

It is a pleasure to acknowledge fruitful conversations with Prof. Grażyna Kontrym-Sznajd.

\section{References}

[1] T.D. Haynes, R.J. Watts, J. Laverock, Zs. Major, M.A. Alam, J.W. Taylor, J.A. Duffy, S.B. Dugdale, New J. Phys. 14, 035020 (2012).

[2] G. Kontrym-Sznajd, Low Temp. Phys. 35, 599 (2009).

[3] G. Kontrym-Sznajd, M. Samsel-Czekała, A. Pietraszko, H. Sormann, S. Manninen, S. Huotari, K. Hämäläinen, J. Laukkanen, R.N. West, W. Schülke, Phys. Rev. B 66, 155110 (2002).

[4] J.P. Carbotte, S. Kahana, Phys. Rev. 139, A213 (1965).

[5] S. Daniuk, G. Kontrym-Sznajd, A. Rubaszek, H. Stachowiak, J. Mayers, P.A. Walters, R.N. West, J. Phys. F 17, 1365 (1987).

[6] H. Sormann, Phys. Rev. B 54, 4558 (1996).

[7] J. Rusz, M. Biasini, A. Czopnik, Phys. Rev. Lett. 93, 156405 (2004).

[8] J. Laverock, T.D. Haynes, M.A. Alam, S.B. Dugdale, Phys. Rev. B 82, 125127 (2010).

[9] A.A. Manuel, D. Vasumathi, B. Barbiellini, A. Shukla, P. Suortti, T. Chiba, Mater. Sci. Forum 255-257, 760 (1997).

[10] H. Sormann, G. Kontrym-Sznajd, Phys. Rev. B 73, 075111 (2006).

[11] G. Kontrym-Sznajd, H. Sormann, E. Boroński, Phys. Rev. B 85, 245104 (2012).

[12] E. Boroński, Nukleonika 55, 3 (2010).

[13] E. Boroński, Mater. Sci. Forum 66 6, 5 (2011).

[14] S. Kahana, Phys. Rev. 129, 1622 (1963).

[15] E. Boroński, Z. Szotek, H. Stachowiak, Phys. Rev. B 23, 1985 (1981).

[16] A. Rubaszek, H. Stachowiak, Phys. Rev. B 38, 3846 (1988).

[17] E. Daniel, S.H. Vosko, Phys. Rev. 120, 2041 (1960).

[18] N.W. Ashcroft, N.D. Mermin, Solid State Physics, Saunders College, Philadelphia 1976.

[19] N.D. Drummond, P. Lopez Rios, R.J. Needs, C.J. Pickard, Phys. Rev. Lett. 107, 207402 (2011). 